\title{
Lateral and anterior thalamic lesions impair independent memory systems
}

\author{
Anna S. Mitchell ${ }^{1,2}$ and John C. Dalrymple-Alford \\ Van der Veer Institute for Parkinson's and Brain Research, and Department of Psychology, University of Canterbury, \\ Christchurch 8020, New Zealand
}

\begin{abstract}
Damage to the medial region of the thalamus, both in clinical cases (e.g., patients with infarcts or the Korsakoff's syndrome) and animal lesion models, is associated with variable amnesic deficits. Some studies suggest that many of these memory deficits rely on the presence of lateral thalamic lesions (LT) that include the intralaminar nuclei, presumably by altering normal function between the striatum and frontal cortex. Other studies suggest that the anterior thalamic nuclei (AT) may be more critical, as a result of disruption to an extended hippocampal system. Here, highly selective LT and AT lesions were made to test the prediction that these two regions contribute to two different memory systems. Only LT lesions produced deficits on a preoperatively acquired response-related (egocentric) working memory task, tested in a cross-maze. Conversely, only AT lesions impaired postoperative acquisition of spatial working memory, tested in a radial maze. These findings provide the first direct evidence of a double dissociation between the LT and AT neural aggregates. As the lateral and the anterior medial thalamus influence parallel independent memory processing systems, they may each contribute to memory deficits, depending on lesion extent in clinical and experimental cases of thalamic amnesia.
\end{abstract}

Profound memory deficits including disruptions to allocentric (place-related) and egocentric (response-related) spatial information processing may occur following medial thalamic injury in humans (Holdstock et al. 1999; Kopelman 2002; Van der Werf et al. 2000, 2003). The source of these impairments is uncertain, with recent reports emphasizing either the intralaminar (ILn) or the anterior (AT) thalamic nuclei (Graff-Radford et al. 1990; Aggleton and Brown 1999; Harding et al. 2000; Mair et al. 2003). Damage to the AT in animal models mimics many of the allocentric spatial memory deficits associated with hippocampal system damage, which is believed to be a key indicator of episodic memory loss (Aggleton and Brown 1999; Aggleton and Pearce 2001; Moran and Dalrymple-Alford 2003; Mitchell and Dalrymple-Alford 2005). Like hippocampal system lesions, AT damage does not generally cause deficits in egocentric responserelated memory tasks (Aggleton et al. 1996; Packard and McGaugh 1996; Warburton et al. 1997; Kesner 1998; Aggleton and Brown 1999; White and McDonald 2002). In contrast, a broader range of deficits is associated with ILn lesions and, coupled with the suggestion that only modest or more selective effects occur after localized AT lesions, this evidence has provided the impetus to suggest that ILn damage is a more essential source of thalamic amnesia (Mair et al. 2003). For example, there are several reports that large ILn lesions produce delay-independent deficits in both spatial and olfactory tasks and evidence that this thalamic region is involved in egocentric response-related processing (Harrison and Mair 1996; Young et al. 1996; Burk and Mair 1998; Mair et al. 1998; Zhang et al. 1998).

The problem with defining the relative roles of the ILn and the AT is that their close proximity often leads to considerable overlap when either region is damaged, both in clinical cases and in animals with experimental lesions. As a result, few studies

\footnotetext{
1Present address: Department of Experimental Psychology, Oxford University, South Parks Road, Oxford, OX1 3UD, UK. ${ }^{2}$ Corresponding author.

E-mail anna.mitchell@psy.ox.ac.uk; fax 44-1865-310447.

Article and publication are at http://www.learnmem.org/cgi/doi/10.1101/ Im.122206.
}

have directly compared highly selective medial thalamus lesions. To address this issue, the current study compared the effects of damage to the lateral medial thalamic region (LT), which included the rostral ILn (comprising the centrolateral, paracentral, and rostral central medial nuclei), and damage to the AT region (comprising the anterodorsal, anteroventral, and anteromedial AT nuclei), using lesion cases in which there was minimal ambiguity in the specificity of the region damaged. The specificity of the LT lesion was guided by the neuroanatomical connections associated with the medial thalamic nuclei, which suggested that the ILn should be grouped together with the adjacent lateral segments of the mediodorsal thalamic nuclei (MD). This LT aggregate collectively has reciprocal connections with overlapping regions of the striatum as well as the anterior cingulate and precentral cortices of the prefrontal cortex (PFC), which thereby form a fronto-striatopallidal-medial thalamic neural circuit (Berendse and Groenewegen 1991; Van der Werf et al. 2002). In contrast, the AT aggregate constitutes a key nodal point within an extended hippocampal system (Shibata 1992, 1998; van Groen and Wyss 1995; Aggleton and Brown 1999; van Groen et al. 1999; Aggleton and Pearce 2001).

With respect to lesions of the LT and the AT regions, there is as yet no clear evidence of any double dissociation, which is necessary to support the suggestion that different thalamic regions may each contribute to parallel independent brain memory systems (Bentivoglio et al. 1997). For example, we recently reported a single dissociation, in that selective AT lesions produced a substantial deficit after preoperative acquisition of a radial-arm maze task, consistent with an influence on an extended hippocampal system, whereas LT lesions produced only weak and transient effects, which may have been due to the small encroachment of some LT lesions into the AT region (Mitchell and Dalrymple-Alford 2005). However, neither lesion impaired postoperative acquisition of working memory for reward value (Mitchell and Dalrymple-Alford 2005). Similarly, the evidence that ILn lesions produce impairments in allocentric spatial memory, reflected by delay-independent deficits in radial-arm maze nonmatching to sample tasks, was based on the effects of 
large ILn lesions that overlapped with the AT region (Burk and Mair 1998; Mair et al. 1998, 2003). Indeed, more restricted ILn lesions have not replicated these findings, even when these lesions were extended to include the mediodorsal nuclei (Bailey and Mair 2005). The aim of the current study was to investigate the possibility of a double dissociation between LT and AT lesions by examining their relative effects on postoperative acquisition of allocentric spatial working memory in the radial maze after testing performance on a preoperatively trained contrast task that depended on response working memory. While only AT lesions were expected to impair spatial memory, the egocentric response task was expected to elicit impairments after damage to the LT region, because lesions to the caudate-putamen and dorsal aspects of the PFC produce deficits in procedural learning, stimulus-response associations, and egocentric localization, but not allocentric spatial location memory (for reviews, see Packard and Knowlton 2002; White and McDonald 2002; Kesner and Rogers 2004).

\section{Results}

\section{Histological analyses}

Figure 1, A and B show the minimum and maximum extent of successful bilateral lesions for the LT and AT groups, respectively. Figure 1, C and D provide photomicrographs of the maximal LT (rat LT201) and AT (rat AT209) lesion cases, respectively. The estimated thalamic damage for each individual rat is shown in Table 1 . In addition to AT and LT damage, data are provided for the posteromedial thalamic region (MT, comprising the central and medial mediodorsal thalamic nuclei and the intermediodorsal nucleus) as this region has also been suggested as a contributor to diencephalic amnesia (Mitchell and Dalrymple-Alford 2005). The criterion of at least $50 \%$ damage to the target region

A

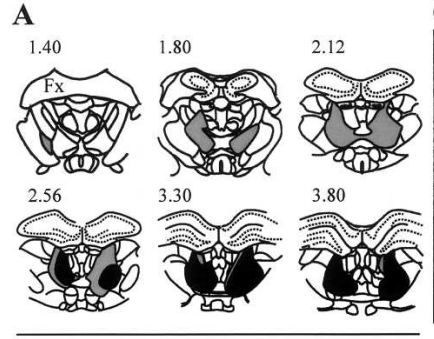

B

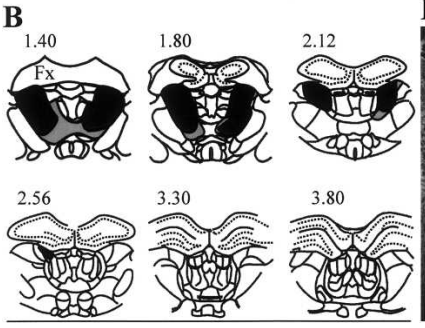

Figure 1. A series of coronal schematics throughout the medial thalamus showing the area of cell loss with the smallest (black) and largest (gray) lesions in $(A)$ the group with lesions to the lateral thalamic aggregate (LT), comprising the intralaminar nuclei (centrolateral, paracentral, and rostral central medial nuclei) and the lateral mediodorsal thalamic nuclei (lateral and paralamellar nuclei) and $(B)$ the group with lesions to the anterior thalamic aggregate (AT), comprising the anterodorsal, anteromedial, and anteroventral thalamic nuclei. Dotted lines represent cell layers in the hippocampus. Numbers in $A$ and $B$ refer to distance posterior to Bregma (reproduced with permission from Elsevier (C) 1998, Paxinos and Watson 1998); (Fx) Fornix. Photomicrographs of the damage evident in the rats with the maximal LT lesion and the maximal AT lesion are shown in C (rat LT201 2.56 in A) and D (rat AT209 1.4 in B), respectively. Note the partial collapse of the AT region in $D$.
(LT or AT) and no more than $40 \%$ damage to nontarget regions (LT, AT, or MT) resulted in the exclusion from the main behavioral analyses of three LT cases (all with excessive MT damage, one of which also had insufficient LT damage) and two AT cases (both with insufficient AT damage). The remaining inclusions $(\mathrm{LT}=6 ; \mathrm{AT}=6)$ resulted in two groups of highly selective lesion cases. The LT inclusions experienced a range of $54.7 \%-76.6 \%$ bilateral damage to the whole LT region itself (combined across the centrolateral, paracentral, and the rostral central medial intralaminar nuclei and the lateral segments of the MD), but little or no AT damage (range, $0.0 \%-21.5 \%$ ) and only modest MT damage (range, $22.5 \%-31.9 \%$ ), although there was generally moderate damage to the adjacent central mediodorsal nucleus (range, $33.9 \%-64.7 \%)$. The central medial nucleus received minor to moderate damage only (range, $0.9 \%-64.4 \%$ ) in these LT cases, but moderate to large damage occurred in the remaining LT components. In terms of other thalamic damage, there was also modest damage to the adjacent ventrolateral nuclei (range, 16.7\%$39.5 \%$ ) in these LT cases. For the accepted AT lesions (range, $78.8 \%-93.6 \%$ ), the adjacent interanteromedial nucleus (range, $4.8 \%-62.5 \%$ ) and paratenial nucleus (range, 2.6\%-39.8\%) were the other nuclei that were generally the most seriously affected. The laterodorsal nucleus, which has also been suggested as an integral part of the anterior thalamic complex (van Groen et al. 1993, 2002b), received little damage in our AT inclusions (range, $1.6 \%-12.9 \%)$. The AT inclusions also experienced relatively little LT damage (range, $7.0 \%-23.9 \%$ ) and minimal MT damage (range, $0.9 \%-3.3 \%$ ).

\section{Behavioral tests}

\section{Memory for egocentric response}

Rats were trained preoperatively until they reached a minimum $75 \%$ correct delayed matching-to-sample (DMS) choices across 10 consecutive preoperative sessions in the response-memory task. As Figure 2A shows, there was no presurgery (Pre) difference in performance between the three randomly assigned groups. After surgery, however, the LT group showed a marked impairment in response working memory that persisted across the first two postoperative blocks of 10 sessions (PST1 and PST2), which used the same 10-sec intratrial delay between the study and test phases as had been used in preoperative training. In contrast, AT lesion rats showed no impairment and were highly comparable to the performance of Controls during the PST1 and PST2 blocks. During the final block of postoperative testing, with a 20-sec intratrial delay (PST3), performance for both the AT and Control groups decreased markedly, while that of the LT group showed no clear additional change. These observations were confirmed by a 3 (between groups) by 4 (Blocks: Pre vs. PST1 vs. PST2 vs. PST3) repeated measures ANOVA, which produced a highly significant group by block interaction, $\left[F_{(6,54)}=4.24, P<0.001\right]$. Analysis of the simple main effects for this interaction showed that the small postoperative reduction in correct DMS choices for the AT and Control groups (PST1 vs. Pre) was not significant, whereas the drop in performance for the LT lesion group was highly significant $(P<0.001)$. For the PST1 block, the LT group was also significantly different from the AT $(P<0.03)$ and Control $(P<0.03)$ groups, which did not differ. All groups improved in the second block of postoperative sessions (PST2), but while the AT and Control groups now showed similar mean performance to their preoperative levels, the LT group remained impaired relative to the Pre block of trials $(P<0.001)$. In the third block (PST3), only the AT and Control groups showed a reduction in correct DMS choices relative to the PST2 block $(P<0.0001$ and $P<0.007$, respectively), whereas there was no significant change across these blocks for the LT group $(P>0.6)$. 
Table 1. Details of medial thalamus lesions in all rats, expressed as percentages of bilateral volumes

\begin{tabular}{|c|c|c|c|c|c|c|c|c|c|c|c|c|c|c|c|c|c|c|c|}
\hline \multirow[b]{2}{*}{$\begin{array}{l}\text { Inclusions/ } \\
\text { exclusions* }\end{array}$} & \multicolumn{4}{|c|}{ AT } & \multicolumn{5}{|c|}{ LT } & \multicolumn{4}{|c|}{ MT } & \multicolumn{6}{|c|}{ Other adjacent nuclei } \\
\hline & $A D$ & AM & AV & $\begin{array}{l}\text { Whole } \\
\text { region }\end{array}$ & $\mathrm{CL}$ & $\begin{array}{l}\text { MDI/ } \\
\text { MDpl }\end{array}$ & PC & rCeM & $\begin{array}{l}\text { Whole } \\
\text { region }\end{array}$ & IMD & MDc & MDm & $\begin{array}{l}\text { Whole } \\
\text { region }\end{array}$ & IAM & LD & PT & $\begin{array}{l}\mathrm{Re} / \\
\mathrm{Rh}\end{array}$ & VL & VN \\
\hline \multicolumn{20}{|c|}{ LT inclusions $(n=6)$} \\
\hline LT203 & 0.0 & 0.0 & 0.0 & 0.0 & 63.2 & 67.6 & 58.1 & 0.9 & 54.7 & 2.0 & 33.9 & 25.8 & 26.3 & 0.0 & 2.3 & 0.0 & 0.0 & 16.7 & 0.3 \\
\hline LT208 & 0.0 & 0.0 & 0.2 & 0.1 & 83.6 & 66.5 & 64.8 & 16.0 & 65.1 & 0.0 & 45.0 & 17.4 & 24.0 & 0.3 & 2.4 & 0.0 & 0.1 & 31.7 & 1.1 \\
\hline LT195 & 0.5 & 3.4 & 1.9 & 2.1 & 77.3 & 85.1 & 59.7 & 13.9 & 66.5 & 0.0 & 49.8 & 13.0 & 22.5 & 0.1 & 1.0 & 0.0 & 0.0 & 24.4 & 0.7 \\
\hline LT183 & 2.0 & 18.9 & 4.2 & 8.6 & 77.6 & 75.9 & 60.2 & 64.4 & 71.1 & 5.3 & 39.5 & 18.2 & 23.3 & 33.6 & 1.5 & 0.0 & 4.1 & 33.4 & 7 \\
\hline LT185 & 0.0 & 4.2 & 2.2 & 2.4 & 80.1 & 82.0 & 68.7 & 37.6 & 72.1 & 3.7 & 49.5 & 24.6 & 30.1 & 0.0 & 1.8 & 0.0 & 0.2 & 39.5 & 0 \\
\hline LT201 & 8.4 & 34.5 & 17.8 & 21.5 & 87.1 & 87.0 & 61.8 & 55.6 & 76.6 & 0.0 & 64.7 & 21.1 & 31.9 & 15.2 & 7.0 & 4.3 & 0.1 & 28.4 & 7.1 \\
\hline LT median & 0.3 & 3.8 & 2.1 & 2.3 & 78.9 & 79.0 & 61.0 & 26.8 & 68.8 & 1.0 & 47.3 & 19.7 & 25.2 & 0.2 & 2.1 & 0.0 & 0.1 & 30.1 & 0.9 \\
\hline \multicolumn{20}{|l|}{ LT exclusions } \\
\hline LT193 & 0.0 & 0.0 & 0.0 & 0.0 & 48.7 & 50.7 & 39.0 & 1.4 & 40.5 & 17.6 & 46.3 & 46.4 & 44.3 & 0.0 & 0.0 & 2.3 & 0.0 & 3.4 & 0 \\
\hline LT207 & 12.7 & 26.1 & 7.6 & 18.6 & 91.4 & 99.1 & 77.1 & 45.1 & 83.7 & 40.8 & 97.6 & 56.0 & 66.7 & 16.9 & 6.0 & 8.0 & 0.3 & 20.0 & 1.0 \\
\hline LT206 & 0.0 & 0.0 & 0.2 & 0.1 & 82.5 & 70.3 & 66.6 & 3.4 & 64.6 & 18.8 & 54.9 & 40.6 & 43.0 & 0.0 & 1.2 & 0.0 & 0.0 & 35.5 & 4.4 \\
\hline \multicolumn{20}{|c|}{ AT inclusions $(n=6)$} \\
\hline AT198 & 88.6 & 35.6 & 96.1 & 78.8 & 10.5 & 1.0 & 12.7 & 0.4 & 7.1 & 0.0 & 0.0 & 2.6 & 1.7 & 4.8 & 11.9 & 9.5 & 0.0 & 0.0 & 0.0 \\
\hline AT190 & 86.7 & 62.4 & 88.1 & 79.4 & 7.0 & 3.0 & 14.5 & 1.5 & 7.0 & 0.0 & 0.0 & 1.3 & 0.9 & 21.9 & 1.6 & 2.6 & 0.0 & 0.0 & 0.0 \\
\hline AT210 & 92.7 & 57.1 & 88.7 & 82.9 & 13.4 & 2.3 & 15.1 & 2.7 & 9.3 & 0.0 & 0.2 & 4.1 & 2.7 & 13.4 & 9.0 & 14.2 & 0.0 & 0.0 & 0.0 \\
\hline AT196 & 83.3 & 64.1 & 92.8 & 86.4 & 7.8 & 3.8 & 19.7 & 3.7 & 9. & 0.0 & 0.0 & 5.2 & 3.3 & 26.4 & 12.9 & 34.9 & 0.0 & 0.0 & 0.0 \\
\hline AT211 & 83.3 & 89.0 & 87.6 & 87.2 & 31.7 & 9.7 & 32.1 & 17.9 & 23.9 & 0.0 & 0.3 & 4.8 & 3.2 & 62.5 & 4.4 & 39.8 & 0.4 & 1.8 & 0.2 \\
\hline АТ209 & 96.6 & 95.2 & 95.9 & 93.6 & 10.7 & 0.2 & 15.5 & 1.8 & 7.8 & 0.0 & 0.0 & 2.2 & 1.4 & 41.9 & 8.1 & 20.7 & 0.8 & 0.1 & 0.0 \\
\hline AT median & 97.7 & 63.3 & 90.8 & 84.7 & 10.6 & 2.7 & 15.3 & 2.3 & 8.5 & 0.0 & 0.0 & 3.4 & 2.2 & 24.2 & 8.6 & 17.5 & 0.0 & 0.0 & 0.0 \\
\hline \multicolumn{20}{|l|}{ AT exclusions } \\
\hline AT199 & 22.0 & 0.7 & 8.4 & 8.5 & 0.0 & 0.0 & 1.5 & 0.0 & 0.4 & 0.0 & 0.0 & 0.1 & 0.1 & 0.0 & 0.0 & 0.6 & 0.0 & 0.0 & 0.0 \\
\hline AT187 & 29.0 & 40.2 & 39.0 & 37.5 & 51.6 & 44.1 & 43.7 & 20.8 & 43.5 & 0.0 & 0.3 & 5.5 & 3.6 & 3.4 & 6.1 & 0.5 & 0.0 & 13.1 & 2.1 \\
\hline
\end{tabular}

*See text for inclusion/exclusion criteria. (AD) Anterodorsal nucleus; (AM) anteromedial nucleus; (AT) anterior thalamic aggregate comprising of the AD, $\mathrm{AM}$, and anteroventral (AV) thalamic nuclei; (AT median) median percentage damage to the individual thalamic nuclei/region of all rats with acceptable AT lesions (AT inclusions); (AT Whole region) percent damage to the AD, AM, and AV combined as a single area; (CL) centrolateral nucleus; (IAM) interanteromedial nucleus; (IMD) intermediodorsal nucleus; (LD) laterodorsal nucleus; (LT) lateral thalamic aggregate comprising the intralaminar nuclei ( $\mathrm{CL}$, paracentral $(\mathrm{PC})$ and rostral central medial $(\mathrm{rCeM})$ nuclei) and lateral mediodorsal thalamic nuclei (lateral (MDI) and paralamellar nuclei $(\mathrm{MDpl})$ ) (LT median) median percentage damage to the individual thalamic nuclei/region of all rats with acceptable LT lesions (LT inclusions); (LT Whole region) percentage damage to the $\mathrm{CL}, \mathrm{PC}, \mathrm{rCeM}, \mathrm{MDI}$, and $\mathrm{MDpl}$, combined as a single area; (MDc) central mediodorsal nucleus; (MDm) medial mediodorsal nucleus; (MT) posteromedial thalamic aggregate comprising the IMD, MDc, MDm; (MT Whole region) damage to the IMD, MDc, and MDm combined as a single area; (PT) parataenial nucleus; (Re/Rh) reuniens nucleus/rhomboid nucleus combined as a single region; (VL) ventrolateral nucleus; (VM) ventromedial nucleus.

\section{Lesion-behavior correlation}

Given the clear lesion-behavior dissociation found in the response-memory task, we considered it appropriate to examine the association between performance in this task and the extent of damage within the medial thalamic targets across all rats with lesions, including those rats that had been excluded from the main between-group behavioral analyses. As the impairment in the LT inclusions was evident across both the first (PST1) and second (PST2) trial blocks, the mean number of correct DMS responses in these two blocks combined was correlated with the extent of damage in the LT aggregate. As shown in Figure 2B, this analysis revealed a strong negative relationship: [Pearson's $r=-0.78, n=9, P<0.013$ ]. Figure $2 \mathrm{~B}$ also shows that the performance of the three excluded LT rats (one primarily a small lesion; two due to excessive encroachment into the MT region) was generally consistent with the extent of their LT lesion. There was no association between correct DMS responses in the first two postoperative blocks and the extent of damage in the AT aggregate: $[r=0.04, n=8$, n.s. (see Figure 2C) .

\section{Spatial working memory}

Postoperative acquisition of the radial-arm maze task was analyzed across five blocks of three trials. As shown in Figure 3A, the number of errors to previously visited arms in the first trial block was equivalent for the three groups, but clear differences emerged between the groups from the second trial block onward. The AT group continued to make numerous arm re-entries across the remaining four blocks of three trials, averaging 8.20 errors per trial (s.d. $=3.39$ ), whereas the LT and Control groups showed comparable performance and steadily decreased their revisit errors from block two onward, such that their average errors fell below 1.0 at the end of training. These observations were confirmed by a 3 (between groups) by 5 (postoperative blocks of three trials) repeated measures ANOVA for the number of revisit errors, which produced a highly significant group effect $\left[F_{(2,18)}=68.18, P<0.0001\right]$, trial block effect $\left[F_{(4,72)}=7.95\right.$, $P<0.0001]$, and group by trial block interaction, $\left[F_{(8,72)}=5.86\right.$, $P<0.0001]$. A repeated measures ANOVA for the number of correct visits made before the first re-entry error (see Fig. 3B) also produced significant effects of group $\left[F_{(2,18)}=45.70, P<0.0001\right]$, trial block $\left[F_{(4,72)}=25.26, P<0.0001\right]$, and group by trial block interaction $\left[F_{(8,72)}=2.64, P<0.02\right]$. Both the LT and Control groups improved at similar rates across trial blocks in terms of arms visited before the first error, but the AT group continued to show a severe deficit on this measure at the end of testing.

As data for the latency to make an arm choice were available for the radial maze task, this measure was also examined to determine whether any of the error scores reflected differences in speed of responding and general activity. There were no significant effects of group or group by trial-block interaction for either correct arm visits $(F<1.0)$ or re-entry visits $(F<1.0)$ across the five blocks of training. Significant effects of trial block for correct arm visits $\left[F_{(4,72)}=7.80, P<0.0001\right]$ and re-entry visits $\left[F_{(4,72)}=5.95\right.$, $P<0.001]$ were found, which reflected the fact that all groups markedly decreased their choice latencies after the first trial block 
A

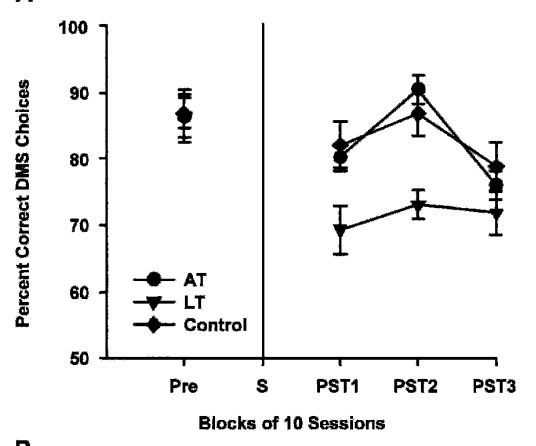

B

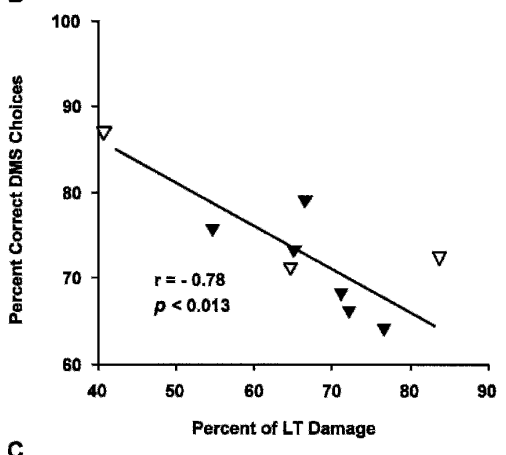

C

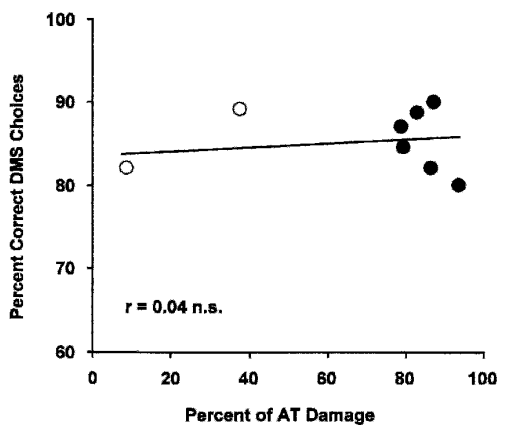

Figure 2. Response-memory task. $(A)$ Percent $( \pm$ SEM) of correct delayed matching-to-sample (DMS) responses made both pre- and postoperatively, grouped into blocks of 10 sessions. The group with lesions to the lateral thalamic aggregate (LT), comprising the intralaminar nuclei (centrolateral, paracentral, and rostral central medial nuclei) and the lateral mediodorsal thalamic nuclei (lateral and paralamellar nuclei), was different than both the controls and the group with lesions to the anterior thalamic aggregate (AT), comprising the anterodorsal, anteromedial, and anteroventral thalamic nuclei, $P<0.001$, during the first two blocks of postoperative testing, when a $10-\mathrm{sec}$ intratrial delay was used (PST1 and PST2); there were no differences when the delay was subsequently increased to $20 \mathrm{sec}$ for the last postoperative session block (PST3). (Pre) Last 10 presurgery training sessions using a $10-\mathrm{sec}$ intratrial delay between study and test phases; (S) surgery. (B) Scattergram of percent damage for all rats with lesions intended for the lateral thalamic aggregate $(L T ; n=9)$ and the percentage number of correct delayed matching-to-sample (DMS) responses combined across PST1 and PST2 blocks of sessions. Solid triangles, LT inclusions $(n=6)$; open triangles, LT exclusions $(n=3)$. (C) Scattergram of percent damage for all rats with lesions intended for the anterior thalamic aggregate (AT; $n=8$ ) and the percentage of correct delayed matching-to-sample (DMS) responses combined across PST1 and PST2 blocks of sessions. Solid circles, AT inclusions $(n=6)$; open circles, AT exclusions $(n=2)$. See text and Table 1 for inclusion/exclusion details.

(data not shown). Across the final three blocks of training, when error scores for the AT group had relatively stabilized, choice latency still produced no significant effect of group, block, or group by trial block interaction for either correct arm visits $(F \mathrm{~s}<1.0)$ or re-entry visits $(F \mathrm{~s}<1.0)$.

\section{Lesion-behavior correlations}

As with the response-memory task, we also examined the association between radial-arm maze task performance and medial thalamic damage across all rats with lesions, including those rats that had been excluded from the main between-group behavioral analyses. The key comparison here is the average performance across the final three blocks of training, because this was when error scores for the AT group had relatively stabilized and all rats were running freely in the maze. As shown in Figure 3C, the AT inclusions clustered together with about seven to nine revisits per trial, whereas the two exclusions made relatively few errors, and this pattern produced a strong positive correlation [Pearson's $r=0.91, n=8, P<0.002]$; the number of arms visited before an error revealed a similar picture $[r=-0.71, n=8, P<0.05$; data not shown]. In contrast, there was no association between the extent of damage in the LT aggregate and either measure for the final three blocks of training [revisit errors, $r=0.55, n=9$, n.s. (see Fig. 3D); arms visited before error: $r=-0.22, n=9$, n.s.].

The corresponding associations between lesion extent and error scores for the first two blocks of training were less clear, probably because many of the rats were not running freely at the start of training. For example, the AT animals made only an average of $7.6 \mathrm{arm}$ visits during the first training trial, 10 visits during the second trial and 12 visits by the third trial. For the first two blocks of training, the extent of AT damage correlated with the number of arms visited before an error, but not with the mean number of revisit errors: [respectively, $r=-0.73, n=8$, $P<0.04$ and $r=0.32, n=8$, n.s.]. Conversely, the extent of LT damage did not correlate with the number of arms visited before an error, but was correlated with the mean number of revisit errors $[r=0.77, n=9, P<0.02$; data not shown]. While this latter association may be a chance result, it is possible that the overall pattern of findings for the LT group reflects a weak transient influence of LT lesions. Nonetheless, there was a clear dissociation in the radial-arm maze task between substantial deficits in the AT group and absent (arm visits before first error), or at best, very minor and transient impairments (revisit errors) in the LT group.

\section{Discussion}

The current study used two different working memory tasks to compare the effects of selective lesions to two aggregates of medial thalamic nuclei, each of which have been implicated in diencephalic amnesia. The LT region included the rostral ILn and the lateral MD, whereas the AT region consisted of the anterodorsal, anteromedial, and anteroventral nuclei. The working memory tasks revealed a novel double dissociation between the effects of damage to these two adjacent thalamic regions. Only lesions to the LT aggregate impaired performance in a preoperatively acquired egocentric response-memory task, tested in the dark in a cross-maze; in this instance, rats with AT lesions performed equivalently to controls. Conversely, only AT lesions impaired postoperative acquisition of the allocentric spatial task, tested under normal light conditions in a radial-arm maze; on this second task, rats with LT lesions were comparable to the controls.

The pattern of spared and impaired memory after LT lesions in the current study is consistent with the conclusions of two recent reports. In one study, Bailey and Mair (2005) found that ILn lesions that spared the AT did not affect a varying choicedelayed nonmatching-to-sample task (up to $25 \mathrm{sec}$ ), which utilizes place-related information for optimal performance and is sensitive to AT and hippocampal lesions (Mair et al. 2003). The same study showed, however, that these restricted ILn lesions impaired performance in an operant-delayed matching-to- 
A

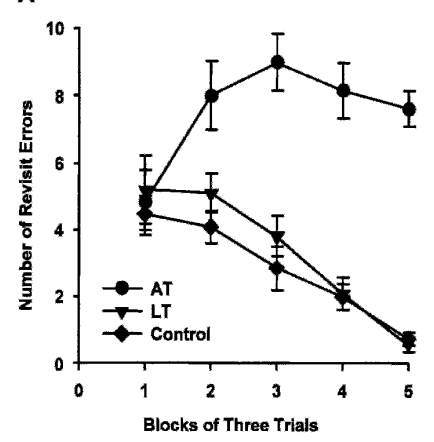

C

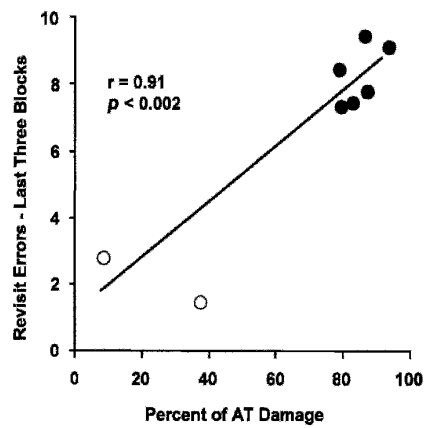

B

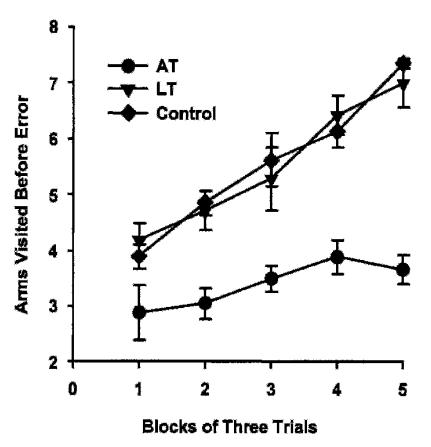

D

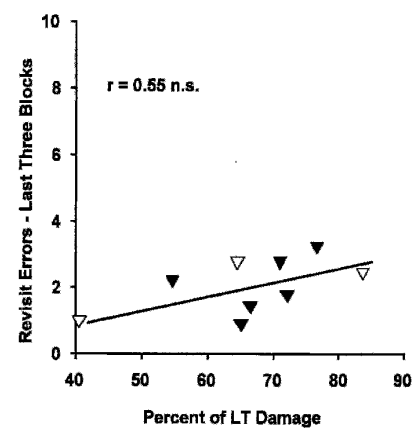

Figure 3. Spatial memory task. Postoperative acquisition of an all-arms baited version of an 8-arm radial maze task. $(A)$ Mean ( \pm SEM) number of revisit errors to the eight baited arms. The group with lesions to the anterior thalamic aggregate (AT), comprising the anterodorsal, anteromedial, and anteroventral thalamic nuclei, was different from both the controls and the group with lesions to the lateral thalamic aggregate (LT), comprising the intralaminar nuclei (centrolateral, paracentral, and rostral central medial nuclei) and the lateral mediodorsal thalamic nuclei (lateral and paralamellar nuclei), $P<0.0001$. ( $B$ ) Mean $( \pm$ SEM) number of correct arm visits made before a revisit error. The AT group was different to both the controls and the LT group, $P<0.0001$. (C) Scattergram of mean percent damage for all rats with lesions intended for the anterior thalamic aggregate (AT; $n=8$ ) and the mean number of revisit errors for the last three blocks of postoperative training trials. Solid circles, AT inclusions $(n=6)$; open circles, AT exclusions $(n=2)$. (D) Scattergram of mean percent damage for all rats with lesions intended for the lateral thalamic aggregate (LT; $n=9)$ and the mean number of revisit errors for the last three blocks of post-operative training trials. Solid triangles, LT inclusions $(n=6)$; open triangles, LT exclusions $(n=3)$. See text and Table 1 for inclusion/exclusion details.

sample task with retractable levers, especially if these lesions included the midline or MD nuclei. This second task is sensitive to prefrontal cortex but not hippocampal lesions (Mair et al. 1998, 2003; Porter et al. 2000). Deficits on both tasks are found when large ILn lesions encroach on the AT region (Burk and Mair 1998; Mair et al. 1998; Savage et al. 1998). Similarly, we have also recently reported that selective lesions to the LT aggregate, which included the ILn and lateral MD, produced only a very mild, transient impairment in a preoperatively trained radial maze task (Mitchell and Dalrymple-Alford 2005). The current study has replicated our previous finding that AT lesions, but not LT lesions, substantially impair performance in the radial-arm maze, and thus extends our previously reported dissociation to the context of postoperative acquisition of radial-arm maze performance. To minimize damage to the adjacent AT region (0\%-22\%; median, $2 \%)$, the LT lesions in the current study were necessarily subtotal (55\%-77\%; median, 69\%) and there was, in particular, relatively minor damage to the rostral central medial nucleus (1\%-64\%; median, $27 \%$ ), so it is possible that more complete LT lesions would have more of an effect on spatial memory. However, it

seems more likely that large LT lesions would only, at best, have a transient effect on acquisition of spatial working memory, and perhaps only in terms of revisit errors. The effectiveness of our LT lesions was nonetheless clear in terms of impairments in the response-memory task, although larger LT lesions were generally associated with more severe memory impairments in this task. The finding that LT lesions were associated with marked deficits for working memory in the response-related task, and not spatial working memory in the radial-arm maze, reinforces the idea of a degree of functional specificity for the LT region (Bailey and Mair 2005).

The DMS egocentric response-memory task, which was modeled on that described by Ragozzino and Kesner (2001), took a considerable period of time (up to 90 sessions) before the rats acquired the preoperative criterion of at least $75 \%$ correct DMS responses across 10 consecutive sessions. This difficulty supports the view that egocentric responses can be readily disrupted or are often transient (Baird et al. 2004), which makes it difficult to use transfer tests such as moving the rat to a different arm in the intratrial interval. Other studies investigating place versus response acquisition using a plus maze in lit conditions have indicated that the level of training may be important to the type of strategy used to solve the task, in addition to the availability of cues. For example, it has been reported that place learning tends to dominate choices early in training, whereas response learning dominates later in training (Packard and McGaugh 1996; Colombo et al. 2003). Rats in the current study may also have relied on a nonegocentric or nonresponse-related strategy to solve this DMS version of the task, such as a sense of room direction, despite the testing being carried out in the dark (Dudchenko and Davidson 2002). For example, during the choice phase they may simply have used intramaze cues to avoid the sample arm that they had most recently exited, despite every attempt to wipe the floor and walls clean after the sample phase, or learned to choose the same direction to that from which they had recently come during the sample phase. Both hippocampal and medial caudate lesions can impair directional learning in rats (DeCoteau et al. 2004), so the possibility of directional learning differences between LT and AT rats is an important alternative to examine in future work on thalamic lesions. While these alternative possibilities may be plausible explanations for how the rats solved the DMS response-memory task, the interesting fact remains that there was a double dissociation between LT and AT lesions and postoperative performance across two behaviorally distinct tasks.

The LT lesions produced deficits only at the short delay in the response-memory task, as the deficit apparent at the 10-sec intratrial delay was not exacerbated by increasing the delay to 20 sec, which was nonetheless effective in reducing performance in controls and AT rats. The performance of the LT rats was, on average, about $70 \%$ at the 10 -sec delay, so their performance was above chance levels prior to the extension of the intratrial delay. Although it seems likely that floor levels of performance would be manifested by chance performance, the current data do not provide evidence that this may be so for the task used. However, the previous study on which the current response-memory task was based did find that rats with dorsal prefrontal cortex injury showed a reduction toward chance levels (Ragozzino and Kesner 2001). It is also possible that the lack of any further deficit in the LT group with the extension of the intratrial delay to $20 \mathrm{sec}$ was due to some degree of recovery of function, although no obvious recovery was apparent in the 10 sessions immediately prior to testing the longer delay. There is, however, other evidence that delay-independent deficits may be a consistent feature across a variety of tasks after both large (Young et al. 1996; Burk and Mair 1998; Zhang et al. 1998) and, now, selective lesions centered on the ILn region (the current findings; Bailey and Mair 2005). Such 
a deficit pattern implicates attentional, rather than memory deficiencies as a consequence of LT lesions, but this seems unlikely as other research is inconsistent with a general attention deficit. For example, slowed response latencies without poor response accuracy has been found in the five-choice serial reaction time task after large ILn lesions, which suggests that ILn lesions may affect the ability to make a voluntary movement to an external stimulus rather than disrupt attentional processes per se (Burk and Mair 2001). Similarly, Newman and Burk (2005) found that ILn lesions only transiently disrupted performance on a sustained attention task and did not increase susceptibility to distracters. Deficits in response selection may provide another explanation for the ILn impairments in the egocentric responserelated task, as it appears that the ILn are involved in activating anatomically interconnected PFC and striatum during periods of behavioral arousal (Groenewegen and Berendse 1994). Judging by our data, small ILn lesions do not seem to have a general influence on response selection or intention, at least in terms of that reflected by-choice latency in the radial arm maze (latency was not collected for the response-memory task). Further studies are required to investigate the generality of response selection impairments after the medial thalamic regions.

The neuroanatomical connections of the LT aggregate indicate that it contributes to a functional circuit that also includes the striatum and the frontal cortex, including a direct influence of the LT aggregate on the spiny cells of the dorsal caudateputamen (Alexander et al. 1986; Berendse and Groenewegen 1991; Burk and Mair 1999; Groenewegen et al. 1999; Haber and McFarland 2001; Mair et al. 2002, 2003; Van der Werf et al. 2002; Bailey and Mair 2005). Lesions to the dorsal caudate-putamen and frontal cortex also produce deficits in response-related memory tasks (Cook and Kesner 1988; Kesner et al. 1993, 1996; McDonald and White 1993; Packard and McGaugh 1996; de Bruin et al. 1997, 2001; Ragozzino and Kesner 2001). Hence, it seems likely that the LT region (i.e., rostral ILn and lateral MD) contribute to a neural circuit that processes information associated with egocentric space and responses or related rules and strategies (White and McDonald 2002). Further evidence to support this proposal can be found in a neurophysiological study of lateral MD neurons in rats, which suggested the existence of a neural circuit involving the anterior cingulate cortex (dorsal medial PFC), striatum, and lateral MD in conditioned motor activity (Oyoshi et al. 1996).

The AT lesion effect on spatial memory in the radial-arm maze task complements a growing list of place-related spatial memory impairments following selective lesions to the AT or its subcomponents (Aggleton et al. 1996; Byatt and DalrympleAlford 1996; van Groen et al. 2002a; Mair et al. 2003; Moran and Dalrymple-Alford 2003; Mitchell and Dalrymple-Alford 2005). The AT mainly influence hippocampal functioning via projections to the pre- and parasubiculum, which in turn project directly to the hippocampus proper or indirectly via the entorhinal cortex; another indirect hippocampal route runs via the retrosplenial cortex (Shibata 1993; van Groen and Wyss 1995; Aggleton and Saunders 1997). Deficits in spatial memory and one-trial memory for object-place associations have also been reported in both rats and monkeys with lesions to either the pre- or parasubiculum or the retrosplenial cortex (Liu et al. 2001; Malkova and Mishkin 2003; Vann and Aggleton 2004a). Hence, there is now convincing evidence of the contribution of the AT within an extended hippocampal circuit that is particularly important for allocentric spatial memory processing in rats and monkeys, often regarded as a crucial aspect of episodic memory (Parker and Gaffan 1997; Aggleton and Brown 1999; Aggleton and Pearce 2001). The specific functional contributions of the AT are uncertain, but different subcomponents of the AT are involved in pro- cessing theta activity and head direction cells, perhaps in concert with the mammillary bodies and Gudden's tegmental nucleus of the brainstem (Blair et al. 1999; Sharp et al. 2001; Vertes et al. 2001; Vann and Aggleton 2004b). AT lesions also inhibit c-fos activity in the retrosplenial cortex and hippocampal system (Jenkins et al. 2002).

The pattern of spared and impaired performance after AT lesions, both in the current study and in previous reports, also agrees with the suggestion that the AT occupy a nodal point in an extended hippocampal memory system (Aggleton and Brown 1999; Aggleton and Pearce 2001). The current finding that AT lesions did not produce deficits in the Ragozzino and Kesner (2001) response-memory task, tested in the dark, is consistent with recent evidence that AT lesions have shown normal acquisition and reversal of an egocentric rule when tested in the presence of visual cues (always turn to the left or to the right) (Aggleton et al. 1996; Warburton et al. 1997) and that neither fornix nor retrosplenial cortex lesions impair working memory for egocentric responses (Baird et al. 2004). Similarly, direct hippocampal damage does not generally impair egocentric or responserelated memory tasks (Kesner 1998; White and McDonald 2002). Previous studies with associative memory tasks provide a more complex, but as yet unexplained, set of results. Both AT and hippocampal lesions, but not fornix lesions, impair the acquisition of an object-in-place task, while only hippocampal lesions impair conditional cue-response associative learning tasks (Sziklas et al. 1998; Sziklas and Petrides 1999, 2002, 2004; Henry et al. 2004). The effects of AT lesions are not, however, restricted to spatial tasks, as they also produce profound deficits when the rat is required to recall the temporal order of a list of odor cues, much the same as hippocampal lesions (Fortin et al. 2002; Kesner et al. 2002; Wolff et al. 2006).

Evidence that memory deficits associated with damage in the medial thalamus are heterogeneous instigated the research question addressed in the current study. The double dissociation between selective LT and AT lesions in the two forms of working memory used here suggests that these two regions may each be important in explaining some of the memory deficits associated with thalamic amnesia. In human infarct cases with ILn damage and in Korsakoff's syndrome cases there is some evidence of deficits in response memory or related tasks (Mair et al. 1998, 2002; Holdstock et al. 1999; Exner et al. 2001). There is also evidence that amnesia is specifically related to damage in the AT and its related pathways (von Cramon et al. 1985; Ghika-Schmid and Bogousslavsky 2000; Harding et al. 2000). Variability and uncertainty across strategically placed lesions is common both in human cases (Parkin et al. 1994; Della Sala et al. 1997; Van der Werf et al. 2000, 2003; Schmahmann 2003) and animal models of diencephalic amnesia. Hence, the severity and extent of deficits observed may reflect lesion size and the number of disparate neural systems affected. Damage that occurs across medial thalamic nuclei and their connections will by this account affect different aspects of memory processing, as each medial thalamic region is predominantly connected with parallel partially independent operating pathways, structures, and cortical regions (Aggleton and Brown 1999; Bailey and Mair 2005; Mitchell and Dalrymple-Alford 2005).

\section{Materials and Methods}

\section{Subjects}

Groups of four female PVGc hooded rats, initial weight 180-220 $\mathrm{gm}$, were housed in opaque plastic cages $(27 \mathrm{~cm} \times 45 \mathrm{~cm}$ wide $\times 22 \mathrm{~cm}$ high) under a reversed light schedule (off 0800 $2000 \mathrm{~h}$ ). Rats had free access to water and were maintained at $80 \%-85 \%$ of ad libitum weight, bar free food access just prior to 
and after surgery to facilitate postoperative recovery. Testing occurred between 0830 and $1930 \mathrm{~h}$ for five sessions per week. All protocols conformed to the NIH Guide for the Care and Use of Laboratory Animals and were approved by the Animal Ethics Committee of the University of Canterbury.

\section{Surgery}

Two groups of rats received either LT $(n=9)$ or AT $(n=8)$ lesions (see Table 2 for details and coordinates). Anesthetized rats (50 $\mathrm{mg} / \mathrm{mL}$ pentobaribitone at $1.65 \mathrm{~mL} / \mathrm{kg}, 20 \mathrm{~min}$ after $0.13 \mathrm{mg} / \mathrm{mL}$ atropine at $1.5 \mathrm{~mL} / \mathrm{kg}$, ip) were placed in a stereotaxic apparatus with the incisor bar set $7.5 \mathrm{~mm}$ below the interaural line to minimize or avoid fornix injury. After craniotomy, microinfusions of $0.12 \mathrm{M} \mathrm{N}$-methyl-D-aspartate (Sigma Chemicals) dissolved in phosphate buffer ( $\mathrm{pH}$ 7.20) were made via a 1- $\mu$ l Hamilton syringe connected to a motorized infusion pump, using $3 \mathrm{~min}$ for diffusion after the infusion at each site. To maximize lesion accuracy, anterior-posterior (AP) coordinates in the horizontal plane were varied according to the Bregma-to-Lambda distance (B-L) in each rat. A third group (Controls, $n=9$ ) received sham lesion surgery but no infusion; the same AP and ML coordinates were used from the lesion groups, spread evenly across Controls, $-0.25 \mathrm{~cm} \mathrm{DV}$ at the corresponding anterior and lateral coordinates.

\section{Behavioral training}

\section{Response working memory task}

\section{Apparatus}

The apparatus and procedure for this task was modeled on that described by Ragozzino and Kesner (2001). A clear Perspex "plus" maze was positioned on a solid table, elevated $70 \mathrm{~cm}$ above the ground. The plus maze had four arms that were $55-\mathrm{cm}$ long with $22-\mathrm{cm}$ high walls. To remove all visual cues and to minimize obvious spatial cues, all testing was conducted with only a red photographic (darkroom) light directly beneath the table for the experimenter (i.e., effectively in the dark for the rats). A curtain surrounded the maze and an infrared camera positioned above the maze relayed recorded images of plus-maze behavior to the adjacent control room.

\section{Procedure}

Rats received presurgery training in the response-memory task and were always carried into the room in an opaque covered cage with the lights out. The rats were familiarized to the plus-maze with 0.1-gm chocolate chip pieces scattered throughout the arms, initially as cage mates and then individually for $5 \mathrm{~d}$ prior to training to criterion in the task using a delayed matching-to-

Table 2. Methodology for N-methyl-D-aspartate lesions of the two medial thalamic aggregates: Coordinates (cm) for various Bregma-Lambda (B-L) measurements, infusion volumes, and rates

\begin{tabular}{|c|c|c|c|c|c|}
\hline & \multicolumn{3}{|c|}{ LT } & \multicolumn{2}{|c|}{ AT } \\
\hline & Anterior & Anterior & Posterior & Anterior & Posterior \\
\hline \multicolumn{6}{|c|}{ AP coordinates for $B-L$ distances $(\mathrm{cm})$} \\
\hline $0.60-0.61$ & -0.345 & -0.345 & -0.385 & -0.245 & -0.255 \\
\hline $0.62-0.63$ & -0.355 & -0.355 & -0.395 & -0.255 & -0.265 \\
\hline $0.64-0.66$ & -0.365 & -0.365 & -0.405 & -0.265 & -0.275 \\
\hline $0.67-0.68$ & -0.375 & -0.375 & -0.415 & -0.275 & -0.285 \\
\hline $\mathrm{ML}$ & \pm 0.130 & \pm 0.130 & \pm 0.130 & \pm 0.123 & \pm 0.147 \\
\hline DV & -0.56 & -0.60 & -0.56 & -0.58 & -0.555 \\
\hline Volume $(\mu \mathrm{l}, 0.12 \mathrm{M})$ & 0.05 & 0.07 & 0.05 & 0.09 & 0.12 \\
\hline Infusion Rate (min) & 2 & 2 & 2 & 3 & 4 \\
\hline
\end{tabular}

(Anterior) Anterior AP coordinates; (AP) anterior-posterior distance from bregma; (AT) anterior thalamic aggregate comprising the anterodorsal, anteromedial, and anteroventral thalamic nuclei; (DV) dorsal-ventral distance from dura; (LT) lateral medial thalamic aggregate comprising the intralaminar nuclei (centrolateral, paracentral, and rostral central medial nuclei) and lateral mediodorsal thalamic nuclei (lateral and paralamellar nuclei; see Van der Werf et al. 2002); (ML) medial-lateral distance from midline; (Posterior) posterior AP site. sample (DMS) procedure with 12 trials per session, five sessions per week. A trial consisted of both a study and a test phase. During the study phase, two clear Perspex blocks were positioned in the arms of the maze to force the rat to make either a left or a right $90^{\circ}$ body turn (six to the right and six to the left per session, using pseudo-randomized sequences from Fellows 1967) and, after the rat reached the end of the arm, it was then rewarded with a chocolate chip $(0.1 \mathrm{gm})$ placed on the floor of the maze. The rat was held in the end of the arm for an intratrial delay of $10 \mathrm{sec}$ by completion of the delay, the test phase began by lifting the block out of the arm. During the test phase, the arm entered in the study phase was used as the start arm and the rat could make a choice of either a $90^{\circ}$ left or right turn on exiting this arm as the Perspex blocks had been rearranged during the delay to form a T-maze. After making a choice, the rat was rewarded $(1 \times 0.1 \mathrm{gm}$ chocolate piece $)$ for matching-to-sample, is, choosing the same body turn that they had been forced to and walls of the maze arms were carefully wiped with a weak solution and then dried, both in the intratrial delay and during the 15 -sec intertrial interval. The experimenter randomly changed positions in the room throughout the trials for each rat.

Rats were trained until they reached a minimum of $75 \%$ which took 90 presurgery sessions. All rats were then matched for preoperative performance and blocks of three rats randomly assigned to one of three groups (LT, AT, or sham-lesion controls). Following $10 \mathrm{~d}$ of postoperative recovery, during which time the $85 \%$ weight reduction was re-established, the rats received 20 sessions with the 10-sec intratrial delay, as per presurgery training, and then a further 10 sessions using a 20 -sec intratrial delay between study and test phases. Choice latencies were not recorded for the egocentric response task.

Once the rats had completed the working memory for egocentric response task, postoperative acquisition of a spatial memory task was assessed using an 8-arm radial maze. The alloAcquisition of spatial working memory

Apparatus

Spatial memory was tested using an elevated $(85 \mathrm{~cm}$ above floor) painted black, and equally spaced aluminum arms $(9 \mathrm{~cm}$ wide $\times 65 \mathrm{~cm}$ long). Each arm had 3-cm-high borders and a single Perspex barrier $(25 \times 20 \mathrm{~cm})$ adjacent to the hub. A black wooden insert $(8.5 \times 5 \times 3 \mathrm{~cm})$ at the end of each arm incorporated a food well $(2 \mathrm{~cm}$ diameter, $1 \mathrm{~cm}$ deep), with $2 \times 0.1 \mathrm{gm}$ pieces of chocolate when baited and food odors provided by inaccessible chocolate located underneath the well at all times. Clear Perspex guillotine doors that could be raised singly or as one unit via overhead cables governed access to the arms. The maze was located in a new, windowless rectangular light-gray colored room with numerous spatial cues (high-contrast posters hung on the walls, a door, computer, desk, chair, and experimenter). The light level was 166 Lux at the center of the maze and 215 Lux at the end of an arm.

\section{Procedure}

Rats were familiarized postoperatively to the radial maze with chocolate chip pieces scattered throughout the arms, initially as cage mates, and then individually across $5 \mathrm{~d}$, followed by one training trial per day for 15 trails. At the beginning of the trial, all of the arms

\section{Learning \& Memory}


were baited with one piece of chocolate chip and the rat was placed in the central hub with all of the doors closed. All doors were then raised simultaneously, allowing the rat a choice of any arm. On entering an arm, all other doors were lowered, followed by the remaining door on the rat's return to the central hub. After a 5-sec delay, all eight doors were raised again allowing another choice, and the trial was complete once the rat had entered all eight baited arms or $10 \mathrm{~min}$ had elapsed. The whole maze was carefully wiped clean with a weak detergent solution between rats.

\section{Histology}

The histological analyses used in this study replicated those used by Mitchell and Dalrymple-Alford (2005). At the completion of the experiment, all rats were transcardially perfused with cold saline followed by $4 \%$ formalin. The brains were removed and post-fixed for $2 \mathrm{~d}$ in $4 \%$ formalin, cryoprotected in 30\% sucrose, and a cryostat used to collect every coronal $50-\mu \mathrm{m}$ section throughout the thalamic region, which were then stained for cell bodies with cresyl violet. Both authors independently estimated the extent of thalamic damage using the relevant plates from a standard rat brain atlas (Paxinos and Watson 1998) and differences were resolved by consensus, but without reference to the behavioral data (only J.D.A. was blind to the individual behavioral data). We chose to make a consensus of the lesions as blinded inter-rater reliability across lesion cases would not ensure greater accuracy, and the clarity of the results obtained points to a satisfactory procedure. The agreed lesion extent was the usual combination of identifying both intact and damaged regions and the area of the lesion was then replicated on electronic copies of the atlas. Automated pixel counting of the percent damage to relevant brain regions in this electronic version was used to generate estimated lesion volumes by factoring in the distances provided by the atlas. This procedure is an elaboration of the conventional "by eye" estimation used in the literature, but explicitly targets the whole medial thalamus region. Collapse of areas surrounding a lesion and variation in angle of sections required a conventional visual, rather than direct image, analysis. Acceptable LT lesions were defined as having more than 50\% bilateral damage to the LT, but not more than $40 \%$ damage to either the adjacent AT or the adjacent MT region. Acceptable AT lesions required more than $50 \%$ damage to this region, but not more than $40 \%$ damage to the LT or MT regions.

\section{Acknowledgments}

A.M. was a recipient of a Foundation of Research, Science and Technology, Top Achiever Doctoral Scholarship through the New Zealand Government. This research was supported by grants from the Department of Psychology, University of Canterbury, Christchurch, New Zealand.

\section{References}

Aggleton, J.P. and Brown, M.W. 1999. Episodic memory, amnesia, and the hippocampal-anterior thalamic axis. Behav. Brain Sci. 22: 425-444. discussion 444-489.

Aggleton, J.P. and Pearce, J.M. 2001. Neural systems underlying episodic memory: Insights from animal research. Philos. Trans. R. Soc. Lond. B Biol. Sci. 356: 1467-1482.

Aggleton, J.P. and Saunders, R.C. 1997. The relationships between temporal lobe and diencephalic structures implicated in anterograde amnesia. Memory 5: 49-71.

Aggleton, J.P., Hunt, P.R., Nagle, S., and Neave, N. 1996. The effects of selective lesions within the anterior thalamic nuclei on spatial memory in the rat. Behav. Brain Res. 81: 189-198.

Alexander, G.E., De Long, M.R., and Strick, P.L. 1986. Parallel organization of functionally segregated circuits linking basal ganglia and cortex. Annu. Rev. Neurosci. 9: 357-381.

Bailey, K.R. and Mair, R.G. 2005. Lesions of specific and nonspecific thalamic nuclei affect prefrontal cortex-dependent aspects of spatial working memory. Behav. Neurosci. 119: 410-419.

Baird, A.L., Futter, J.E., Muir, J.L., and Aggleton, J.P. 2004. On the transience of egocentric working memory: Evidence from testing the contribution of limbic brain regions. Behav. Neurosci. 118: 785-797.
Bentivoglio, M., Aggleton, J.P., and Mishkin, M. 1997. The thalamus and memory formation. In Thalamus: Experimental and clinical aspects (eds. M. Steriade and E.J. Jones), Vol. 2, pp. 689-721. Elsevier, Amsterdam, The Netherlands.

Berendse, H.W. and Groenewegen, H.J. 1991. Restricted cortical termination fields of the midline and intralaminar thalamic nuclei in the rat. Neuroscience 42: 73-102.

Blair, H.T., Cho, J., and Sharp, P.E. 1999. The anterior thalamic head-direction signal is abolished by bilateral but not unilateral lesions of the lateral mammillary nucleus. J. Neurosci. 19: 6673-6683.

Burk, J.A. and Mair, R.G. 1998. Thalamic amnesia reconsidered: Excitotoxic lesions of the intralaminar nuclei, but not the mediodorsal nucleus, disrupt place delayed matching-to-sample performance in rats (Rattus norvegicus). Behav. Neurosci. 112: 54-67. . 1999. Delayed matching-to-sample trained with retractable levers is impaired by lesions of the intralaminar or ventromedial but not the laterodorsal thalamic nuclei. Psychobiology 27: 351-363. . 2001. Effects of intralaminar thalamic lesions on sensory attention and motor intention in the rat: A comparison with lesions involving frontal cortex and hippocampus. Behav. Brain Res. 123: 49-63.

Byatt, G. and Dalrymple-Alford, J.C. 1996. Both anteromedial and anteroventral thalamic lesions impair radial-maze learning in rats. Behav. Neurosci. 110: 1335-1348.

Colombo, P.J., Brightwell, J.J., and Countryman, R.A. 2003. Cognitive strategy-specific increases in phosphorylated cAMP response element-binding protein and c-Fos in the hippocampus and dorsal striatum. J. Neurosci. 23: 3547-3554.

Cook, D. and Kesner, R.P. 1988. Caudate nucleus and memory for egocentric localization. Behav. Neural Biol. 49: 332-343.

de Bruin, J.P., Swinkels, W.A., and de Brabander, J.M. 1997. Response learning of rats in a Morris water maze: Involvement of the medial prefrontal cortex. Behav. Brain Res. 85: 47-55.

de Bruin, J.P., Moita, M.P., de Brabander, H.M., and Joosten, R.N. 2001. Place and response learning of rats in a Morris water maze: Differential effects of fimbria fornix and medial prefrontal cortex lesions. Neurobiol. Learn. Mem. 75: 164-178.

DeCoteau, W.E., Hoang, L., Huff, L., Stone, A., and Kesner, R.P. 2004. Effects of hippocampus and medial caudate nucleus lesions on memory for direction information in rats. Behav. Neurosci. 118: $540-545$.

Della Sala, S., Spinnler, H., and Venneri, A. 1997. Persistent global amnesia following right thalamic stroke: An 11-year longitudinal study. Neuropsychology 11: 90-103.

Dudchenko, P.A. and Davidson, M. 2002. Rats use a sense of direction to alternate on T-mazes located in adjacent rooms. Anim. Cogn. 5: $115-118$.

Exner, C., Weniger, G., and Irle, E. 2001. Implicit and explicit memory after focal thalamic lesions. Neurology 57: 2054-2063.

Fellows, B.J. 1967. Chance stimulus sequences for discrimination tasks. Psychol. Bull. 67: 87-92.

Fortin, N.J., Agster, K.L., and Eichenbaum, H.B. 2002. Critical role of the hippocampus in memory for sequences of events. Nat. Neurosci. 5: $458-462$.

Ghika-Schmid, F. and Bogousslavsky, J. 2000. The acute behavioral syndrome of anterior thalamic infarction: A prospective study of 12 cases. Ann. Neurol. 48: 220-227.

Graff-Radford, N.R., Tranel, D., van Hoesen, G.W., and Brandt, J.P. 1990. Diencephalic amnesia. Brain 113: $1-25$.

Groenewegen, H.J. and Berendse, H.W. 1994. The specificity of the 'non-specific' midline and intralaminar thalamic nuclei. Trends Neurosci. 17: 52-57.

Groenewegen, H.J., Galis-de Graaf, Y., and Smeets, W.J. 1999. Integration and segregation of limbic cortico-striatal loops at the thalamic level: An experimental tracing study in rats. J. Chem. Neuroanat. 16: 167-185.

Haber, S. and McFarland, N.R. 2001. The place of the thalamus in frontal cortical-basal ganglia circuits. Neuroscientist 7: 315-324.

Harding, A., Halliday, G., Caine, D., and Kril, J. 2000. Degeneration of anterior thalamic nuclei differentiates alcoholics with amnesia. Brain 123: $141-154$.

Harrison, L.M. and Mair, R.G. 1996. A comparison of the effects of frontal cortical and thalamic lesions on measures of spatial learning and memory in the rat. Behav. Brain Res. 75: 195-206.

Henry, J., Petrides, M., St-Laurent, M., and Sziklas, V. 2004. Spatial conditional associative learning: Effects of thalamo-hippocampal disconnection in rats. Neuroreport 15: 2427-2431.

Holdstock, J.S., Mayes, A.R., Cezayirili, E., Isaac, C.L., Aggleton, J.P., and Roberts, N. 1999. A comparison of egocentric and allocentric spatial memory in medial temporal lobe and Korsakoff's amnesics. Cortex 35: $479-501$. 
Jenkins, T.A., Dias, R., Amin, E., Brown, M.W., and Aggleton, J.P. 2002 Fos imaging reveals that lesions of the anterior thalamic nuclei produce widespread limbic hypoactivity in rats. J. Neurosci. 22: 5230-5238.

Kesner, R.P. 1998. Neurobiological views of memory. In Neurobiology of Learning and Memory. (eds. J.L. Martinez and R.P. Kesner), pp. 361-416. Academic Press, San Diego, CA.

Kesner, R.P. and Rogers, J. 2004. An analysis of independence and interactions of brain substrates that subserve multiple attributes, memory systems, and underlying processes. Neurobiol. Learn. Mem. 82: 199-215

Kesner, R.P., Bolland, B.L., and Dakis, M. 1993. Memory for spatial locations, motor responses, and objects: Triple dissociation among the hippocampus, caudate nucleus, and extrastriate visual cortex. Exp. Brain Res. 93: 462-470.

Kesner, R.P., Hunt, M.E., Williams, J.M., and Long, J.M. 1996. Prefrontal cortex and working memory for spatial response, spatial location, and visual object information in the rat. Cereb. Cortex 6: 311-318.

Kesner, R.P., Gilbert, P.E., and Barua, L.A. 2002. The role of the hippocampus in memory for the temporal order of a sequence of odors. Behav. Neurosci. 116: 286-290.

Kopelman, M.D. 2002. Disorders of memory. Brain 125: 2152-2190.

Liu, P., Jarrard, L.E., and Bilkey, D.K. 2001. Excitotoxic lesions of the pre- and parasubiculum disrupt object recognition and spatial memory processes. Behav. Neurosci. 115: 112-124.

Mair, R.G., Burk, J.A., and Porter, M.C. 1998. Lesions of the frontal cortex, hippocampus, and intralaminar thalamic nuclei have distinct effects on remembering in rats. Behav. Neurosci. 112: 772-792.

Mair, R.G., Koch, J.K., Newman, J.B., Howard, J.R., and Burk, J.A. 2002. A double dissociation within striatum between serial reaction time and radial maze delayed nonmatching performance in rats. $J$. Neurosci. 22: 6756-6765.

Mair, R.G., Burk, J.A., and Porter, M.C. 2003. Impairment of radial maze delayed nonmatching after lesions of anterior thalamus and parahippocampal cortex. Behav. Neurosci. 117: 596-605.

Malkova, L. and Mishkin, M. 2003. One-trial memory for object-place associations after separate lesions of hippocampus and posterior parahippocampal region in monkey. J. Neurosci. 23: 1956-1965.

McDonald, R.J. and White, N.M. 1993. A triple dissociation of memory systems: Hippocampus, amygdala, and dorsal striatum. Behav. Neurosci. 107: 3-22.

Mitchell, A.S. and Dalrymple-Alford, J.C. 2005. Dissociable memory effects after medial thalamus lesions. Eur. J. Neurosci. 22: 973-985.

Moran, J.P. and Dalrymple-Alford, J.C. 2003. Perirhinal cortex and anterior thalamic lesions: Comparative effects on learning and memory. Behav. Neurosci. 117: 1326-1341.

Newman, L.A. and Burk, J.A. 2005. Effects of excitotoxic thalamic intralaminar nuclei lesions on attention and working memory. Behav. Brain Res. 162: 264-271.

Oyoshi, T., Nishijo, H., Asakura, T., Takamura, Y., and Ono, T. 1996. Emotional and behavioral correlates of mediodorsal thalamic neurons during associative learning in rats. J. Neurosci. 16: $5812-5829$.

Packard, M.G. and Knowlton, B.J. 2002. Learning and memory functions of the Basal Ganglia. Annu. Rev. Neurosci. 25: 563-593.

Packard, M.G. and McGaugh, J.L. 1996. Inactivation of hippocampus or caudate nucleus with lidocaine differentially affects expression of place and response learning. Neurobiol. Learn. Mem. 65: 65-72.

Parker, A. and Gaffan, D. 1997. The effect of anterior thalamic and cingulate cortex lesions on object-in-place memory in monkeys. Neuropsychologia 35: 1093-1102.

Parkin, A.J., Rees, J.E., Hunkin, N.M., and Rose, P.E. 1994. Impairment of memory following discrete thalamic infarction. Neuropsychologia 32: $39-51$.

Paxinos, G. and Watson, C. 1998. The rat brain in stereotaxic coordinates, 4th ed. Academic Press, San Diego, CA

Porter, M.C., Burk, J.A., and Mair, R.G. 2000. A comparison of the effects of hippocampal or prefrontal cortical lesions on three versions of delayed non-matching-to-sample based on positional or spatial cues. Behav. Brain Res. 109: 69-81.

Ragozzino, M. and Kesner, R.P. 2001. The role of rat dorsomedial prefrontal cortex in working memory for egocentric responses. Neurosci. Lett. 308: 145-148.

Savage, L.M., Castillo, R., and Langlais, P.J. 1998. Effects of lesions of thalamic intralaminar and midline nuclei and internal medullary lamina on spatial memory and object discrimination. Behav. Neurosci. 112: 1339-1352.

Schmahmann, J.D. 2003. Vascular syndromes of the thalamus. Stroke 34: 2264-2278.
Sharp, P.E., Blair, H.T., and Cho, J. 2001. The anatomical and computational basis of the rat head-direction cell signal. Trends Neurosci. 24: 289-294.

Shibata, H. 1992. Topographic organization of subcortical projections to the anterior thalamic nuclei in the rat. J. Comp. Neurol.

323: 117-127.

-1993. Direct projections from the anterior thalamic nuclei to the retrohippocampal region in the rat. J. Comp. Neurol. 337: 431-445. . 1998. Organization of projections of rat retrosplenial cortex to the anterior thalamic nuclei. Eur. J. Neurosci. 10: 3210-3219.

Sziklas, V. and Petrides, M. 1999. The effects of lesions to the anterior thalamic nuclei on object-place associations in rats. Eur. J. Neurosci. 11: $559-566$.

. 2002. Effects of lesions to the hippocampus or the fornix on allocentric conditional associative learning in rats. Hippocampus 12: $543-550$.

- 2004. Egocentric conditional associative learning: Effects of restricted lesions to the hippocampo-mammillo-thalamic pathway. Hippocampus 14: 931-934.

Sziklas, V., Lebel, S., and Petrides, M. 1998. Conditional associative learning and the hippocampal system. Hippocampus 8: 131-137.

Van der Werf, Y.D., Witter, M.P., Uylings, H.B., and Jolles, J. 2000. Neuropsychology of infarctions in the thalamus: A review. Neuropsychologia 38: 613-627.

Van der Werf, Y.D., Witter, M.P., and Groenewegen, H.J. 2002. The intralaminar and midline nuclei of the thalamus. Anatomical and functional evidence for participation in processes of arousal and awareness. Brain Res. Brain Res. Rev. 39: 107-140.

Van der Werf, Y.D., Scheltens, P., Lindeboom, J., Witter, M.P., Uylings, H.B.M., and Jolles, J. 2003. Deficits of memory, executive functioning and attention following infarction in the thalamus; a study of 22 cases with localised lesions. Neuropsychologia 41: $1330-1344$

van Groen, T. and Wyss, J.M. 1995. Projections from the anterodorsal and anteroventral nucleus of the thalamus to the limbic cortex in the rat. J. Comp. Neurol. 358: 584-604.

van Groen, T., Vogt, B.A., and Wyss, J.M. 1993. Interconnections between the thalamus and the retrosplenial cortex in rodent brain. In The neurobiology of the cingulate cortex and limbic thalamus. (eds. B.A. Vogt and M. Gabriel), pp. 123-150. Birkhauser, Boston, MA.

van Groen, T., Kadish, I., and Wyss, J.M. 1999. Efferent connections of the anteromedial nucleus of the thalamus of the rat. Brain Res. Brain Res. Rev. 30: 1-26.

- 2002a. Role of the anterodorsal and anteroventral nuclei of the thalamus in spatial memory in the rat. Behav. Brain Res. 132: 19-28.

- 2002b. The role of the laterodorsal nucleus of the thalamus in spatial learning and memory in the rat. Behav. Brain Res. 136: 329-337.

Vann, S.D. and Aggleton, J.P. 2004a. Testing the importance of the retrosplenial guidance system: Effects of different sized retrosplenial cortex lesions on heading direction and spatial working memory. Behav. Brain Res. 155: 97-108.

. 2004b. The mammillary bodies: Two memory systems in one? Nat. Rev. Neurosci. 5: 35-44.

Vertes, R.P., Albo, Z., and Viana Di Prisco, G. 2001. Theta-rhythmically firing neurons in the anterior thalamus: Implications for mnemonic functions of Papez's circuit. Neuroscience 104: 619-625.

von Cramon, D.Y., Hebel, N., and Schuri, U. 1985. A contribution to the anatomical basis of thalamic amnesia. Brain 108: 993-1008.

Warburton, E.C., Baird, A.L., and Aggleton, J.P. 1997. Assessing the magnitude of the allocentric spatial deficit associated with complete loss of the anterior thalamic nuclei in rats. Behav. Brain Res. 87: 223-232.

White, N.M. and McDonald, R.J. 2002. Multiple parallel memory systems in the brain of the rat. Neurobiol. Learn. Mem. 77: 125-184

Wolff, M., Gibb, S.J., and Dalrymple-Alford, J.C. 2006. Beyond spatial memory: The anterior thalamus and memory for the temporal order of a sequence of odour cues. J. Neurosci. 26: 2907-2913.

Young, H.L., Stevens, A.A., Converse, E., and Mair, R.G. 1996. A comparison of temporal decay in place memory tasks in rats (Rattus norvegicus) with lesions affecting thalamus, frontal cortex, or the hippocampal system. Behav. Neurosci. 110: 1244-1260.

Zhang, Y.P., Burk, J.A., Glode, B.M., and Mair, R.G. 1998. The effects of thalamic and olfactory cortical lesions on continuous olfactory DNMTS and olfactory discrimination in the rat. Behav. Neurosci. 112: $39-53$.

Received November 17, 2005; accepted in revised form February 23, 2006. 


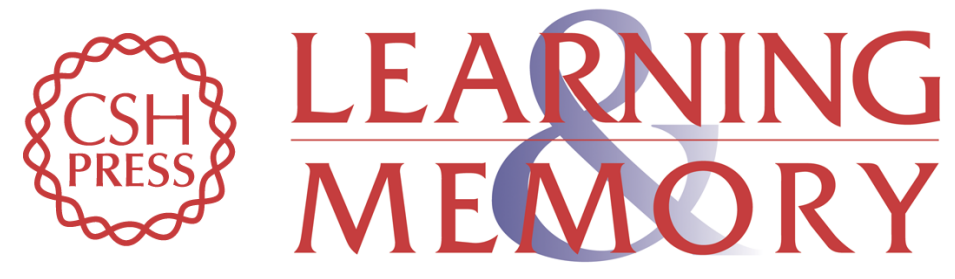

\section{Lateral and anterior thalamic lesions impair independent memory systems}

Anna S. Mitchell and John C. Dalrymple-Alford

Learn. Mem. 2006, 13:

Access the most recent version at doi:10.1101//m.122206

References This article cites 80 articles, 9 of which can be accessed free at: http://learnmem.cshlp.org/content/13/3/388.full.html\#ref-list-1

License

Email Alerting

Receive free email alerts when new articles cite this article - sign up in the box at the Service top right corner of the article or click here. 